\title{
Nitrofurantoin-induced lung disease and prophylaxis of urinary tract infections
}

\author{
*Yasser Madania, Bhupinder Mann ${ }^{b}$ \\ ${ }^{a}$ Core Trainee 2 Doctor, Department of Respiratory Medicine, West Middlesex University Hospital, London, UK \\ ${ }^{b}$ Consultant Respiratory Physician, Department of Respiratory Medicine, West Middlesex University Hospital, London, UK
}

Originally received 23rd February 2012; resubmitted 11th April 2012; revised 29th April 2012; accepted 6th May 2012; online 26th July 2012

\begin{abstract}
Summary
Nitrofurantoin is commonly used for the treatment and prophylaxis of recurrent urinary tract infections (UTIs). Although relatively rare, nitrofurantoin is one of the commonest causes of drug-induced pulmonary disease, which can be potentially serious and even fatal. Knowledge of such potential adverse effects is essential to enable early recognition and withdrawal of the drug. Patients on long-term nitrofurantoin should be reviewed and monitored regularly. Management involves early consideration of the condition, and prompt withdrawal of the drug. We report three cases of nitrofurantoin-induced lung disease in patients who were on long-term nitrofurantoin for UTI prophylaxis and present a brief review of the literature on this subject.

(c) 2012 Primary Care Respiratory Society UK. All rights reserved.

Y Madani and B Mann. Prim Care Respir J 2012; 21(3): 337-341

http://dx.doi.org/10.4104/pcrj.2012.00059
\end{abstract}

Keywords nitrofurantoin, lung disease, drug-induced, fibrosis, urinary tract infection, prophylaxis

\section{Introduction}

Nitrofurantoin has been commonly used for more than 50 years for the treatment and prophylaxis of recurrent urinary tract infections (UTIS). Potential adverse effects include pulmonary toxicity, hepatitis, cholestasis, peripheral neuropathy and aplastic anaemia.' Pulmonary toxicity can be either acute or chronic. Although relatively uncommon, occurring in less than $1 \%$ of patients, nitrofurantoin is one of the commonest causes of drug-induced pulmonary disease, with potentially serious and fatal outcomes. ${ }^{2,3}$

\section{Case 1}

An 88-year-old woman presented with a 6-month history of a productive cough and progressive dyspnoea with a reduction in exercise tolerance down to 30 metres. There was no history of smoking or occupational exposure. Her past medical history included posterior repair for stress incontinence and recurrent UTIs. She had been taking nitrofurantoin for UTI prophylaxis for the past four years. On examination, oxygen saturations were $87-93 \%$ on air and respiratory rate was $17 / \mathrm{min}$. Auscultation of the chest revealed fine inspiratory crepitations throughout. Arterial blood gas ( $A B G$ ) on air showed pH 7.44, $\mathrm{PaCO}_{2} 5.6 \mathrm{kPa}$ and $\mathrm{PaO}_{2} 8.5 \mathrm{kPa}$. Chest $\mathrm{x}$-ray (CXR) showed reticulonodular changes bilaterally. Eosinophil count was elevated at $0.92 \times 10^{\circ} / \mathrm{L}$. Computed tomography (CT) scan of the

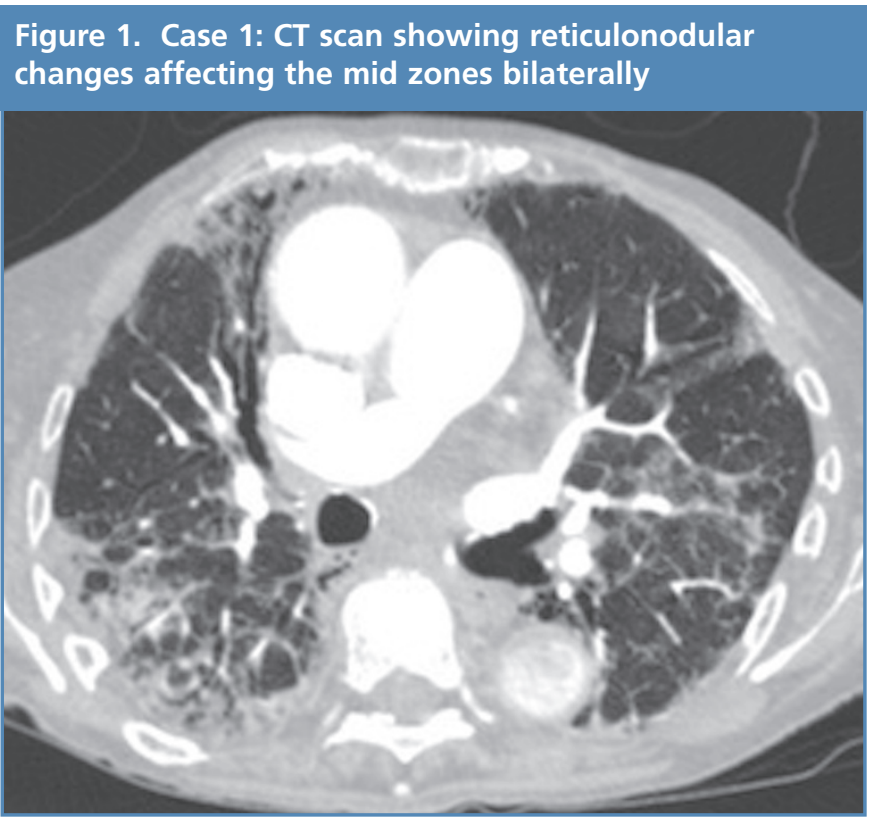

thorax revealed extensive fibrotic changes (reticulonodular opacities) mainly involving the mid and lower zones bilaterally with associated traction bronchiectasis (see Figure 1). Pulmonary function test (PFT)

\footnotetext{
* Corresponding author: Dr Yasser Madani, Department of Respiratory Medicine, West Middlesex University Hospital, Isleworth, Middlesex, London, TW7 6AF, UK. Tel: +44 (0)2085602121 ext 5337 Fax: +44 (0)208 3215581 E-mail: yasser.madani@doctors.org.uk
} 
data was unavailable. She was diagnosed with interstitial lung disease secondary to nitrofurantoin. The nitrofurantoin was stopped, and she had a good response to prednisolone.

\section{Case 2}

An 84-year-old man presented with a 2-month history of a productive cough and dyspnoea causing reduced exercise tolerance from 1.5 kilometres down to 15 metres. He had lost $7 \mathrm{~kg}$ in weight. His past medical history included removal of left renal calculi, bladder diverticulum and urethral stricture treated with meatoplasty with subsequent recurrent UTIs and once-weekly selfcatheterisation. He had been taking prophylactic nitrofurantoin for the past nine years. He was an ex-smoker with a 35 pack-year history. He had no relevant occupational exposures. On examination, oxygen saturations were $90 \%$ on air and respiratory rate was 20/min. He appeared cachectic. Auscultation of the chest revealed crepitations to the midzones bilaterally.

$A B G$ on air showed pH 7.46, $\mathrm{PaCO}_{2} 4.65 \mathrm{kPa}$ and $\mathrm{PaO}_{2} 7.02$ $\mathrm{kPa}$. CXR demonstrated extensive reticular shadowing in both lungs consistent with fibrosis (see Figure 2). High-resolution CT (HRCT) scan of the thorax revealed general emphysematous changes and bibasal and subpleural thickening of the intra-lobular septae, associated with some ground glass opacity and peribronchial cuffing. There were also small bilateral pleural effusions (see Figure 3). PFT revealed forced vital capacity (FVC) 1.73L (50.4\% predicted), forced expiratory volume in one second $\left(\mathrm{FEV}_{1}\right) 1.21 \mathrm{~L}(68.8 \%$ predicted), $\mathrm{FEV}_{1} / \mathrm{FVC}$ ratio 0.7 , residual volume (RV) $143.11 \%$, total lung capacity (TLC) $86.43 \%$, transfer factor of the lung for carbon monoxide (TLCO) 19\% and transfer coefficient for carbon monoxide

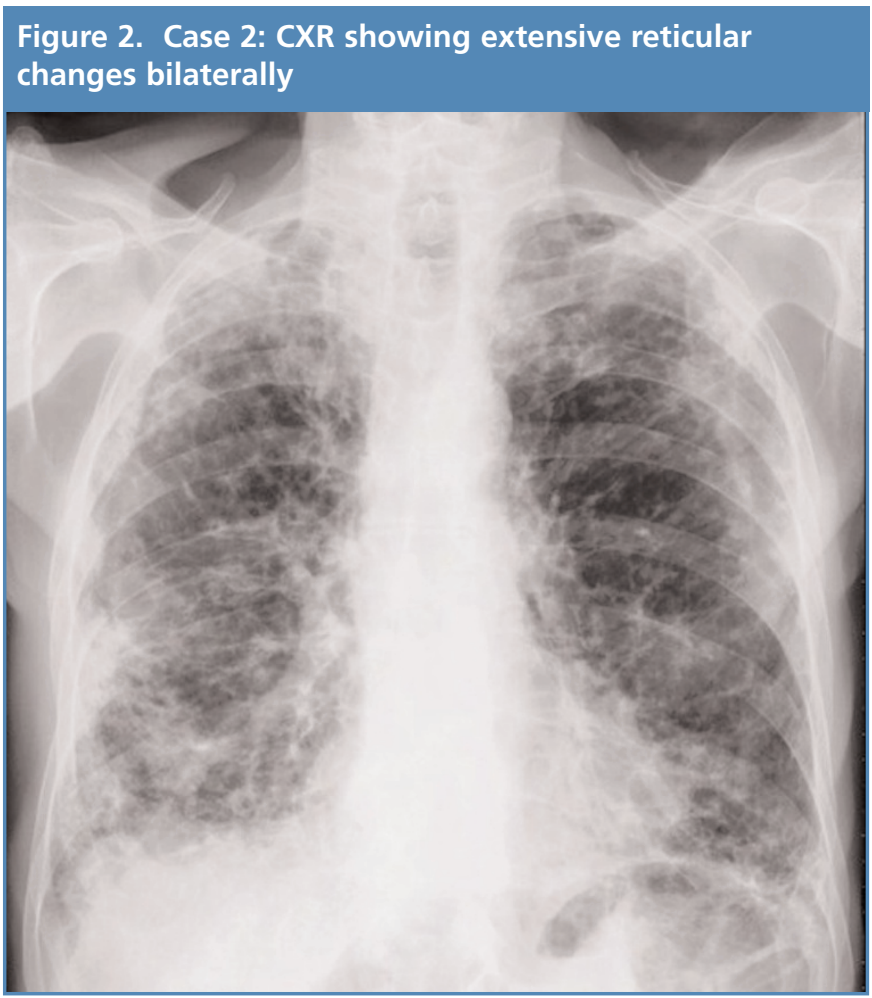

Figure 3. Case 2: HRCT showing bibasal and subpleural intra-lobular septal thickening, with some groundglass opacity and small bilateral pleural effusions

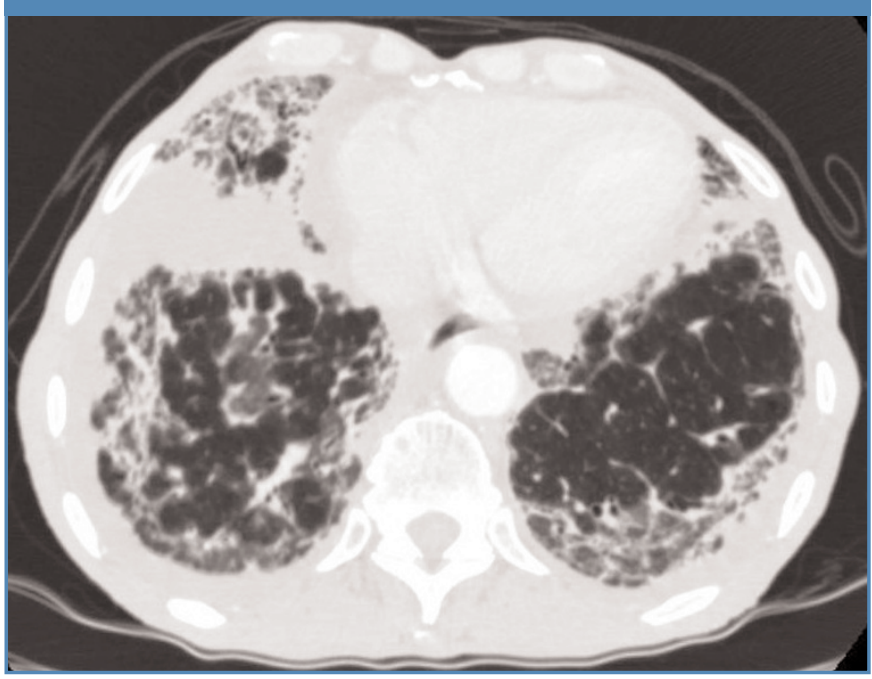

(KCO) $28 \%$. Nitrofurantoin was suspected to be the cause and was discontinued. He was prescribed home oxygen and started on prednisolone. Three months later there was significant improvement in his exercise tolerance and resting oxygen saturations. Objective improvement was seen when he had repeat PFT which showed FVC 3.0L (87.8\%), TLCO 55.7\% and KCO $77.8 \%$.

\section{Case 3}

A 58-year-old woman presented with a 6-month history of cough and dyspnoea with reduction in exercise tolerance from almost $1 \mathrm{~km}$ down to 30 metres. She was a non-smoker and had no occupational exposure of note. Her past medical history included right-sided ureteric stent insertion for hydronephrosis and subsequent UTIs for which she had been taking prophylactic nitrofurantoin for the past seven years. On examination, respiratory rate was 20-25/min and there were fine mid-inspiratory crepitations at both bases and axillae. Her oxygen saturations reduced from $94 \%$ to $84 \%$ after walking approximately 60 yards.

$A B G$ on air showed $\mathrm{PaO}_{2} 9.31 \mathrm{kPa}$ and $\mathrm{PaCO}_{2}$ 4.90kPa. CXR showed reticular changes in both bases and midzones (see Figure 4). CT scan revealed areas of organising pneumonia with fibrosis in a diffuse perilobular pattern, and areas of ground glass changes with traction bronchiectasis. PFT revealed FVC 1.41 (51\%), FEV 1.24 (53\%), FEV $/$ FVC ratio 0.88 , TLC $48 \%$ and TLCO $34 \%$. Echocardiogram revealed a pulmonary arterial pressure (PAP) of $37 \mathrm{mmHg}$. Bronchoalveolar lavage (BAL) revealed a neutrophilia of $10 \%$ and an eosinophilia of $19.7 \%$, which was suggestive of lung injury caused by nitrofurantoin. The nitrofurantoin was therefore stopped. At the same time she was also diagnosed as having undifferentiated connective tissue disease (CTD). Despite treatment with prednisolone, and subsequently azathioprine, cyclophosphamide and mycophenolate, her PFT continued to decline and she eventually required long-term oxygen therapy and died five years after her initial presentation. There was a degree of 


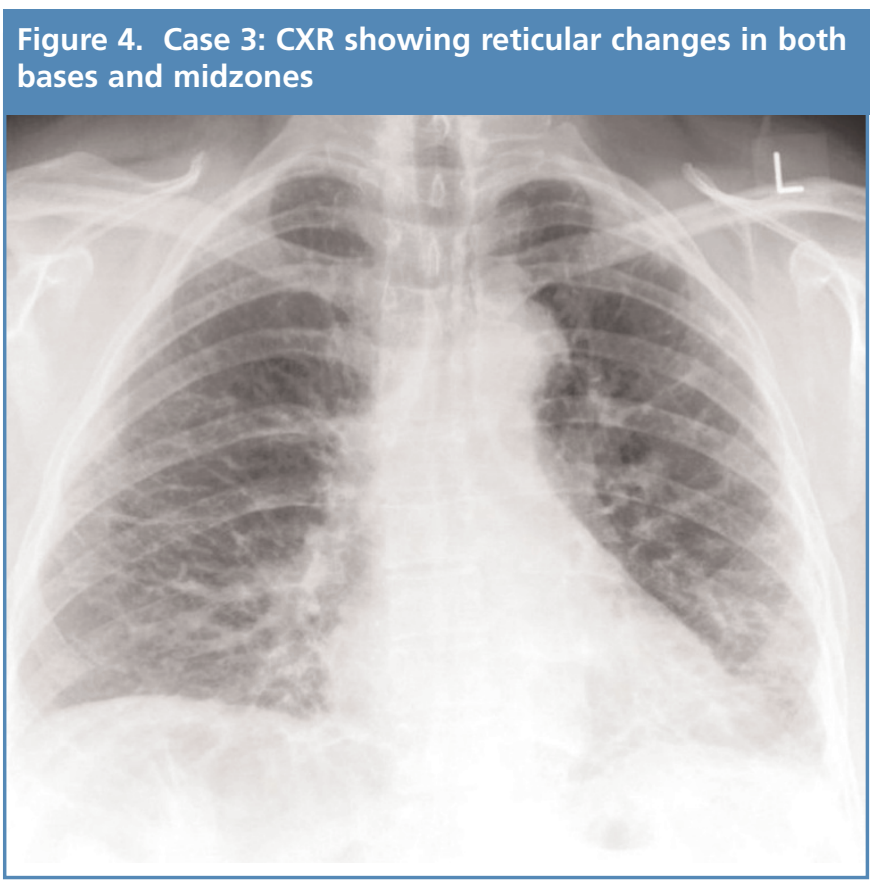

uncertainty about the contribution of the undifferentiated CTD to the final outcome. It was thought that the CTD might well have contributed to her respiratory illness by potentiating the drug toxicity, but the adverse outcome is also more typical of a fibrotic non-specific interstitial pneumonia.

\section{Discussion}

\section{Acute nitrofurantoin-induced lung disease (NILD)}

NILD was first reported by Fisk in 1957 who described an acute reaction. ${ }^{4}$ Rosenow et al. later reported cases of chronic NILD in 1968. ${ }^{5}$ The acute form is far more common and is characterised by cough, dyspnoea, chest pain, fever, rash, arthralgia, tachycardia and tachypnoea. CXR shows reticular shadowing or alveolar infiltrates. Eosinophilia may be present in the serum or in BAL, but is a nonspecific finding and is merely suggestive. PFT may demonstrate a restrictive pattern and reduced diffusion capacity. Symptoms may appear within hours of starting treatment, but often occur nine days after first exposure and much quicker after the second exposure. Symptoms usually resolve after discontinuation of the drug, but failure to resolve within 72 hours should alert the clinician to consider alternative diagnoses such as heart failure, infection and acute respiratory distress syndrome. Acute NILD is thought to be due to a hypersensitivity or immune-mediated reaction. ${ }^{6-9}$

\section{Chronic NILD}

Chronic NILD is 10-20 times less frequent than the acute form, and is not thought to follow on from acute reactions. Similarly, acute reactions do not predispose to a chronic pattern. ${ }^{2,10}$ Chronic NILD is thought to appear up to six months, or even years, after initiation of treatment. ${ }^{1,6,9}$ It is thought to be due to direct toxicity, which, unlike acute hypersensitivity reactions, is dose-related. ${ }^{2,11}$ it is most commonly seen in older women, likely due to their higher prevalence of UTIs, with a median age of 60 to 70 years. ${ }^{2,3}$ It usually
Table 1. Pulmonary manifestations of nitrofurantoininduced lung disease ${ }^{11-16}$

- Pulmonary fibrosis

- Bronchiolitis obliterans organising pneumonia

- Diffuse alveolar damage

- Desquamative interstitial pneumonia

- Nonspecific interstitial pneumonia

- Acute and subacute interstitial pneumonia

- Giant cell interstitial pneumonia

- Eosinophilic pneumonia

- Diffuse alveolar haemorrhage

- Lupus-like syndrome

- Allergic reactions including anaphylaxis

- Acute bronchospasm

- Pleural effusion

presents with an insidious onset of cough and progressive dyspnoea, although chest pain, myalgia, weight loss and fatigue may also occur. ${ }^{11,12}$ Given the insidious onset, symptoms can be mistaken for more common causes such as heart failure. Examination findings include inspiratory crepitations and possibly clubbing. ${ }^{12}$ Eosinophilic pleural effusions may occur. CXR commonly reveals bilateral interstitial infiltrates, either diffusely or predominantly in the lower zones. ${ }^{12}$ PFT may reveal a restrictive pattern with reduced diffusion capacity. ${ }^{11}$ HRCT scan findings are varied and include ground-glass attenuation, honeycombing, inter- and intralobular septal thickening, traction bronchiectasis and organising pneumonia. ${ }^{11-13}$ There are many different forms of pulmonary involvement in NILD (see Table 1).

\section{Investigations}

The most important investigations used to investigate NILD in the hospital setting are shown in Table 2.

\section{Treatment}

The mainstay of treatment is discontinuation of nitrofurantoin. Evidence for the use of corticosteroids is lacking and largely supported by anecdotal case reports. ${ }^{12,13}$ Most centres do not use steroids unless there is severe systemic illness and infection has been excluded as the cause of the symptoms.

\section{Prognosis}

Complete or partial resolution of chronic NILD has been reported both with and without corticosteroids. ${ }^{9,12}$ Most patients report symptomatic improvement on cessation of nitrofurantoin, accompanied by improvement in the radiological abnormalities. Mendez et al. found that all 18 cases of chronic NILD in their series improved symptomatically on discontinuation of nitrofurantoin, with 16 patients improving within two months. Objective evidence of improvement (PFT, CXR and CT) was observed in all but two patients, although 12 had residual infiltrates on follow-up CXR or CT scans. ${ }^{12}$ Chronic interstitial changes on $C T$ can resolve with time without treatment if the drug is stopped; however, it is not uncommon for them to persist long-term. Resolution of chronic 
Table 2. Investigations

\section{Arterial Blood Gas (ABG) Sampling}

$A B G$ are used in the assessment of respiratory function:

- $\mathrm{PaO}_{2}$ is used to assess oxygenation ( $>12 \mathrm{kPa},>10 \mathrm{kPa}$ in the elderly)

- $\mathrm{PaCO}_{2}$ is used in the evaluation of ventilation (4.6-6.0kPa)

- In interstitial lung disease, as in NILD, reduced $\mathrm{PaO}_{2}$ (hypoxia) can be seen.

\section{Pulmonary Function Testing}

i) Gas transfer (diffusion capacity)

- Measures the amount of surface area available for gas transfer.

- A gas mixture containing carbon monoxide (CO) is inhaled, held for 10 seconds and then exhaled.

- The amount of CO that has disappeared (by crossing the alveoli and into pulmonary capillary blood) is measured. A correction is made for the haemoglobin.

- The total amount of CO transferred is termed the Transfer factor of the lung for carbon monoxide (TLCO). When this is divided by the total lung volume during the breath hold it is called the Transfer coefficient ( $\mathrm{kCO}$ ), gas transfer per unit lung volume.

- It is used in the assessment of the severity of interstitial lung disease, as in NILD, where TLCO and KCO are reduced.

i) Lung volumes

- Total lung capacity (TLC) is the total volume in the lungs at maximal inflation.

- Residual volume (RV) is the volume of air remaining in the lungs after a maximal exhalation.

- This is also used in the assessment of interstitial lung disease, where the TLC \& RV are reduced.

\section{High-resolution Computed Tomography (HRCT) Scanning}

- Essential in the diagnosis of interstitial lung disease. The high resolution is brought about by narrow slice width $(1 \mathrm{~mm})$, spacing $(10-20 \mathrm{~mm})$ between image slices, image reconstruction algorithm and minimising the field of view, so as to reduce the size of each pixel.

- HRCT scan findings in interstitial lung disease include:

i) Ground-glass attenuation refers to the hazy increase in lung density which indicates partial displacement of air.

ii) Interlobular septal thickening - interlobular septa surround each pulmonary lobule and contain connective tissue, venules and lymphatics.

iii) Reticular pattern refers to the presence of multiple interlacing lines producing a net-like pattern.

iv) Honeycombing is defined by the presence of small cystic spaces with irregularly thickened walls of fibrous tissue.

NILD can take from several months to a year. ${ }^{5,12}$ Prognosis depends on early recognition and cessation of the drug. In addition, several reports have highlighted deaths due to NILD. ${ }^{2,3,17}$

\section{Use of nitrofurantoin prophylaxis for UTIs}

A Cochrane systematic review of prophylactic antibiotic use for recurrent UTI in non-pregnant women showed that antibiotic prophylaxis reduced rates of recurrent UTI compared to placebo. ${ }^{18}$ This benefit ceased as soon as antibiotics were stopped. The commonest side effects experienced in the treatment group included vaginal and oral candidiasis and gastrointestinal symptoms. Nitrofurantoin had the same risk of adverse events but was associated with more withdrawals from the studies due to these.

\section{Box 1 - Learning Points}

- Nitrofurantoin can cause potentially serious and even fatal pulmonary reactions

- The use of nitrofurantoin should be limited to 6 months unless benefits outweigh the risks

- Patients on long-term nitrofurantoin should be reviewed and monitored regularly

- Nitrofurantoin should be withdrawn at the first sign of pulmonary damage

- Patients should have 6-monthly CXR and monitoring of liver function

The optimal duration of prophylaxis remains unclear, as studies have only looked at prophylaxis for up to 12 months. However, the Society of Obstetricians and Gynaecologists of Canada recommends antibiotic prophylaxis in women with two or more UTIs in six months, or three or more UTIs in 12 months. ${ }^{19}$ In the UK, the Health Protection Agency advocates the use of prophylactic nitrofurantoin or trimethoprim in primary care in women who have had three or more UTIs per year. ${ }^{20}$

Knowledge of the potential adverse effects of nitrofurantoin, especially pulmonary toxicity, is essential to enable early recognition and withdrawal of the drug. It is recommended that the use of prophylactic nitrofurantoin should be limited to six months unless benefits outweigh the risks. ${ }^{17}$ Patients on long-term nitrofurantoin should be reviewed and monitored regularly with regards to complications, and nitrofurantoin should be withdrawn at the first sign of pulmonary damage. This may be indicated by the presence of cough or dyspnoea. Patients should have 6-monthly CXR and monitoring of liver function to allow early detection of complications.11,21 It may be advisable to avoid long-term nitrofurantoin in those with chronic pulmonary conditions given that they are likely to have poor respiratory reserve to begin with. In such cases, trimethoprim may be used as first line.

\section{Handling editor Hilary Pinnock}

Conflicts of interest The authors declare that they have no conflicts of interest in relation to this article.

Contributorship YM developed the idea for writing the manuscript, performed the literature search and wrote the manuscript. BM diagnosed and managed two of the reported cases and reviewed the manuscript.

Funding No financial assistance was sought or provided for the writing of this manuscript.

\section{References}

1. Israel KS, Brashear RE, Sharma HM, Yum MN, Glover JL. Pulmonary fibrosis and nitrofurantoin. Am Rev Respir Dis 1973;108(2):353-6.

2. Holmberg L, Boman G, Bottiger LE, Eriksson B, Spross R, Wessling A. Adverse reactions to nitrofurantoin. Analysis of 921 reports. Am J Med 1980;69:733-8. http://dx.doi.org/10.1016/0002-9343(80)90443-X

3. Holmberg L, Boman G. Pulmonary reactions to nitrofurantoin. 447 cases reported to the Swedish Adverse Drug Reaction Committee 1966-1976. Eur J Respir Dis 1981;62:180-9.

4. Fisk AA. Brief recording: anaphylactoid reaction to nitrofurantoin. N Engl J Med 1957;256(22):1054. http://dx.doi.org/10.1056/NEJM195705302562207 
5. Rosenow EC 3rd, DeRemee RA, Dines DE. Chronic nitrofurantoin pulmonary reaction. Report of 5 cases. N Engl J Med 1968;279(23):1258-62. http://dx.doi.org/10.1056/NEJM196812052792304

6. Hainer BL, White AA: Nitrofurantoin pulmonary toxicity. J Fam Pract 1981;13(6):817-23.

7. Boggess KA, Benedetti TJ, Raghu G: Nitrofurantoin-induced pulmonary toxicity during pregnancy: a report of a case and review of the literature. Obstet Gynecol Surv 1996;51(6):367-70. http://dx.doi.org/10.1097/00006254-199606000-00021

8. Witten CM. Pulmonary toxicity of nitrofurantoin. Arch Phys Med Rehabil 1989;70(1):55-7.

9. Krause M, Ruef C. Miscellaneous antibacterial drugs. In: Dukes MNG, Aronson KH, ed. Meyler's Side Effects of Drugs. 14th ed. Amsterdam: Elsevier BV, 2000. p.884-5.

10. Bhullar S, Lele SM, Kraman S. Severe nitrofurantoin lung disease resolving without the use of steroids. J Postgrad Med 2007;53(2):111-13. http://dx.doi.org/10.4103/0022-3859.32211

11. Martins RR, Marchiori E, Viana SL, Grillo Júnior LS, Capelozzi VL, Valença LM. Chronic eosinophilic pneumonia secondary to long-term use of nitrofurantoin: highresolution computed tomography findings. J Bras Pneumol 2008;34(3):181-4. http://dx.doi.org/10.1590/S1806-37132008000300009

12. Mendez JL, Nadrous HF, Hartman TE, Ryu JH. Chronic nitrofurantoin-induced lung disease. Mayo Clin Proc 2005;80(10):1298-302. http://dx.doi.org/10.4065/80.10.1298

13. Goemaere NN, Grijm K, van Hal PT, den Bakker MA. Nitrofurantoin-induced pulmonary fibrosis: a case report. J Med Case Reports 2008;21(2):169. http://dx.doi.org/10.1186/1752-1947-2-169
14. Willcox PA, Maze SS, Sandler M, Benatar SR. Pulmonary fibrosis following long-term nitrofurantoin therapy. S Afr Med J 1982;61(19):714-17.

15. Cameron RJ, Kolbe J, Wilsher ML, Lambie N. Bronchiolitis obliterans organising pneumonia associated with the use of nitrofurantoin. Thorax 2000;55:249-251. http://dx.doi.org/10.1136/thorax.55.3.249

16. Travis WD, Colby TV, Koss MN, Rosado-de-Christenson ML, Muller NL, King TE. NonNeoplastic Disorders of the Lower Respiratory Tract. In: Atlas of Nontumor Pathology Volume 2. Washington: The American Registry of Pathology, 2002. p.327.

17. Tatley M, Centre for Adverse Reactions Monitoring, Dunedin, New Zealand. (2002). Prescriber Update Articles: Pulmonary Reactions with Nitrofurantoin [online]. MEDSAFE 2002;23(2)24-5. Available: http://www.medsafe.govt.nz/profs/ PUarticles/nitrofurant.htm [Accessed 2012, February 20]

18. Albert X, Huertas I, Pereiró II, Sanfélix J, Gosalbes V, Perrota C. Antibiotics for preventing recurrent urinary tract infection in non-pregnant women. Cochrane Database Syst Rev 2004;(3):CD001209. http://dx.doi.org/10.1002/14651858.CD001209.pub2

19. Epp A, Larochelle A, Lovatsis D, et al. Society of Obstetricians and Gynaecologists of Canada. Recurrent urinary tract infection. J Obstet Gynaecol Can 2010;32(11):1082-101.

20. Health Protection Agency. Management of infection guidance for primary care for consultation and local adaptation [online]. (2010). Available: http://www.hpa.org.uk/webc/HPAwebFile/HPAweb_C/1279888711402 [Accessed 2012, February 20].

21. Parker G. Failure to adequately monitor liver and lung function. MDU J 2007;23: 24-5.

\section{Available online at http://www.thepcrj.org}

